\title{
Cost, safety, and rehabilitation of same- stage, bilateral total knee replacements compared to two-stage total knee replacements
}

\author{
Raymond C. W. Wan ${ }^{1 \dagger}$, Jason C. H. Fan ${ }^{2 *+}$, Yuk-Wah Hung ${ }^{2}$, Ka-Bon Kwok' ${ }^{2}$ Carmen K. M. Lo ${ }^{2}$ and
} Kwong-Yin Chung ${ }^{1}$

\begin{abstract}
Background: Many patients experience bilateral knee osteoarthritis and require bilateral total knee replacement (TKR). Same-stage, bilateral TKR is proposed to be a cost-effective and safe solution compared to two-stage, but conflicting results in the literature are reported. We aim to compare the costs, safety, and rehabilitation performance of patients in same-stage versus two-stage, bilateral TKR with our centre's perioperative protocol.

Materials and methods: We retrospectively reviewed 175 patients (95 same-stage, 80 two-stage) who had undergone bilateral TKR in our centre. Patient selection for same-stage, bilateral TKR was strictly protocol-driven and required fulfilment of all criteria, including age $<75$ years, American Society of Anesthesiologists (ASA) grade 1 or 2 , body mass index $(\mathrm{BMI})<40$, and having non-complex arthritis. All patients followed a standardised pre-operative, intra-operative, and post-operative Enhanced Recovery After Surgery (ERAS) protocol. The cost, safety profiles, and rehabilitation outcomes were compared between the same-stage and two-stage groups.

Results: The same-stage, bilateral TKR reduced the length of hospital stays by 5.71 days per patient, decreased the operation time by $27.4 \mathrm{~min}$, saved 3.34 (18.6\%) physiotherapy sessions, and 3.78 (51.5\%) occupational therapy sessions. The same-stage group experienced a higher haemoglobin drop but no significant difference in transfusion percentage, transfusion volume, complication rate, and readmission rate. The two-stage subgroup with anaesthetic risk, age, and BMI similar to the same-stage group showed the same results. Same-stage, bilateral TKR patients experienced no significant difference in final post-operative pain levels and rehabilitation outcomes as two-stage TKR patients.
\end{abstract}

Conclusion: This study showed that same-stage, bilateral TKR can reduce costs, with similar safety profiles and rehabilitation outcomes compared to the two-stage, bilateral TKR.

Keywords: Knee osteoarthritis, Total knee replacement, Safety, Cost, Rehabilitation outcome

\footnotetext{
* Correspondence: fchjason@gmail.com

${ }^{\dagger}$ Raymond C. W. Wan and Jason C. H. Fan contributed equally to this work.

${ }^{2}$ Present address: Department of Orthopedics \& Traumatology, Alice Ho Miu Ling Nethersole Hospital, Tai Po, Hong Kong SAR, China

Full list of author information is available at the end of the article
}

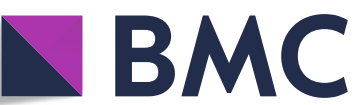

Part of Springer Nature
(9) The Author(s). 2021 Open Access This article is licensed under a Creative Commons Attribution 4.0 International License, which permits use, sharing, adaptation, distribution and reproduction in any medium or format, as long as you give appropriate credit to the original author(s) and the source, provide a link to the Creative Commons licence, and indicate if changes were made. The images or other third party material in this article are included in the article's Creative Commons licence, unless indicated otherwise in a credit line to the material. If material is not included in the article's Creative Commons licence and your intended use is not permitted by statutory regulation or exceeds the permitted use, you will need to obtain permission directly from the copyright holder. To view a copy of this licence, visit http://creativecommons.org/licenses/by/4.0/ The Creative Commons Public Domain Dedication waiver (http://creativecommons.org/publicdomain/zero/1.0/) applies to the data made available in this article, unless otherwise stated in a credit line to the data. 


\section{Introduction}

Knee osteoarthritis is a prevalent disease globally, that affects many patients. In the United States, $12 \%$ of adults are affected by knee osteoarthritis, and total knee replacements (TKRs) cost USD10.2 billion USD annually [1]. Similarly, $8.1 \%$ of the Chinese population is affected by symptomatic knee osteoarthritis [2]. Bilateral knee osteoarthritis is common in that as many as $34.1 \%$ female and $17.5 \%$ male Chinese individuals aged over 65 years suffer from bilateral radiographic knee osteoarthritis [3]. The demand for bilateral TKR is high, leading to a massive healthcare burden. Therefore, a safe and more cost-effective solution is required.

Bilateral knee replacements can be performed either in a same-stage (both knees in the same anaesthetic session) or two-stage (two replacements on two separate operations that can be weeks or months apart) surgical procedure. The advantages of a same-stage, bilateral TKRs include one anaesthetic session, single theatre use, and a single hospital admission, and shorter hospital stay [4-6]. Multiple studies reported that same-stage, bilateral TKR is a safe procedure $[2,7,8]$, with similar morbidities and mortality [9]. The disadvantages include the possibility of more significant post-operative pain, increased rate of cardiovascular events, thromboembolism, blood loss, and mortality $[3,10]$. In addition, some studies reported lower overall cost to the healthcare system $[4,6]$, while others showed no overall cost reduction $[5,11]$. There are also conflicting reports with regards to rehabilitation outcomes $[9,12]$. Some studies are outdated while modern practice may improve outcomes significantly. Overall, the net outcome of same-stage, bilateral TKR-whether it is beneficial to patients in terms of safety and rehabilitation and whether it is beneficial to the healthcare system in terms of cost-effectiveness-remains a controversial topic.

Since 2016, the surgeons at the Joint Replacement Centre (JRC) of Alice Ho Miu Ling Nethersole Hospital in Hong Kong have performed same-stage, bilateral TKR procedures with a standardised protocol of Enhanced Recovery After Surgery (ERAS). This study aims to compare the cost, safety profile, and clinical outcomes of a same-stage, bilateral TKR procedure versus a two-stage, bilateral TKR with patients undergoing the same ERAS protocol. We hypothesized that same-stage, bilateral TKR would reduce cost with similar safety profile and clinical outcomes compared to two-stage, bilateral TKR.

\section{Materials and methods}

\section{Patient selection protocol and defining the patient cohorts}

This retrospective review studied data from all patients with bilateral TKR performed between January 2016 and December 2017 at the JRC in Alice Ho Miu Ling Nethersole Hospital, Hong Kong SAR, China. All patients with bilateral primary TKR performed for bilateral knee osteoarthritis were included. The reviewed patients were segregated into two cohorts: same-stage, bilateral TKR and two-stage, bilateral TKR, according to the surgery that they ultimately received (as treated).

In our centre, patient selection for same-stage, bilateral TKR was strictly protocol-driven. For patients undergoing same-stage, bilateral TKR, they had to fulfil all of the following criteria:

a) Age $<75$ years

b) American Society of Anesthesiologists (ASA) class 1 or 2 according to the anaesthetists' documentation

c) Absence of medical comorbidity, including obstructive sleep apnoea, body mass index (BMI) > 40 (because of the extended operation time and complexity of the procedure) and no history of acute coronary syndrome

d) The knee does not require complex reconstruction by corrective osteotomy

If the patients agreed, they were enrolled for the samestage, bilateral TKR procedure. If the patients disagreed, the reasons were recorded, and a two-stage, bilateral TKR procedure was performed.

We identified a total of 175 patients. The Consolidated Standards of Reporting Trials (CONSORT) flow diagram is shown in Fig. 1. Among them, 95 patients (26 male and 69 female) received a same-stage TKR and 80 patients (22 male, 58 female) received a two-stage, bilateral TKR. Twenty-two patients fit the inclusion criteria for the same-stage, bilateral TKR but were selected for the twostage, bilateral TKR group for the following reasons: 20 (90.9\%) patients preferred the two-stage procedure; one (4.5\%) case was related to a caring plan issue; and one (4.5\%) patient had initially been prepared for the samestage, bilateral TKR, but the second knee surgery was abandoned intra-operatively due to an injury to the popliteal artery of the first knee.

Only one patient who did not fit the inclusion criteria, a 77-year-old man with good past health and having ASA 1, received the same-stage surgery; he expressed a very strong preference for same-stage, bilateral TKR.

Institutional Review Board ethics approval was obtained from the Chinese University of Hong Kong, Hong Kong SAR, CRE Ref. No. 2019.250 (Submission Ref. No. NTEC-2019-0093). Informed consent is not required as this is a retrospective review of medical records which contain no identifiable information of the patient.

\section{Perioperative protocol - ERAS protocol Pre-operation}

Before the operation, structured education classes were provided by JRC specialty nurses, physiotherapists, and 


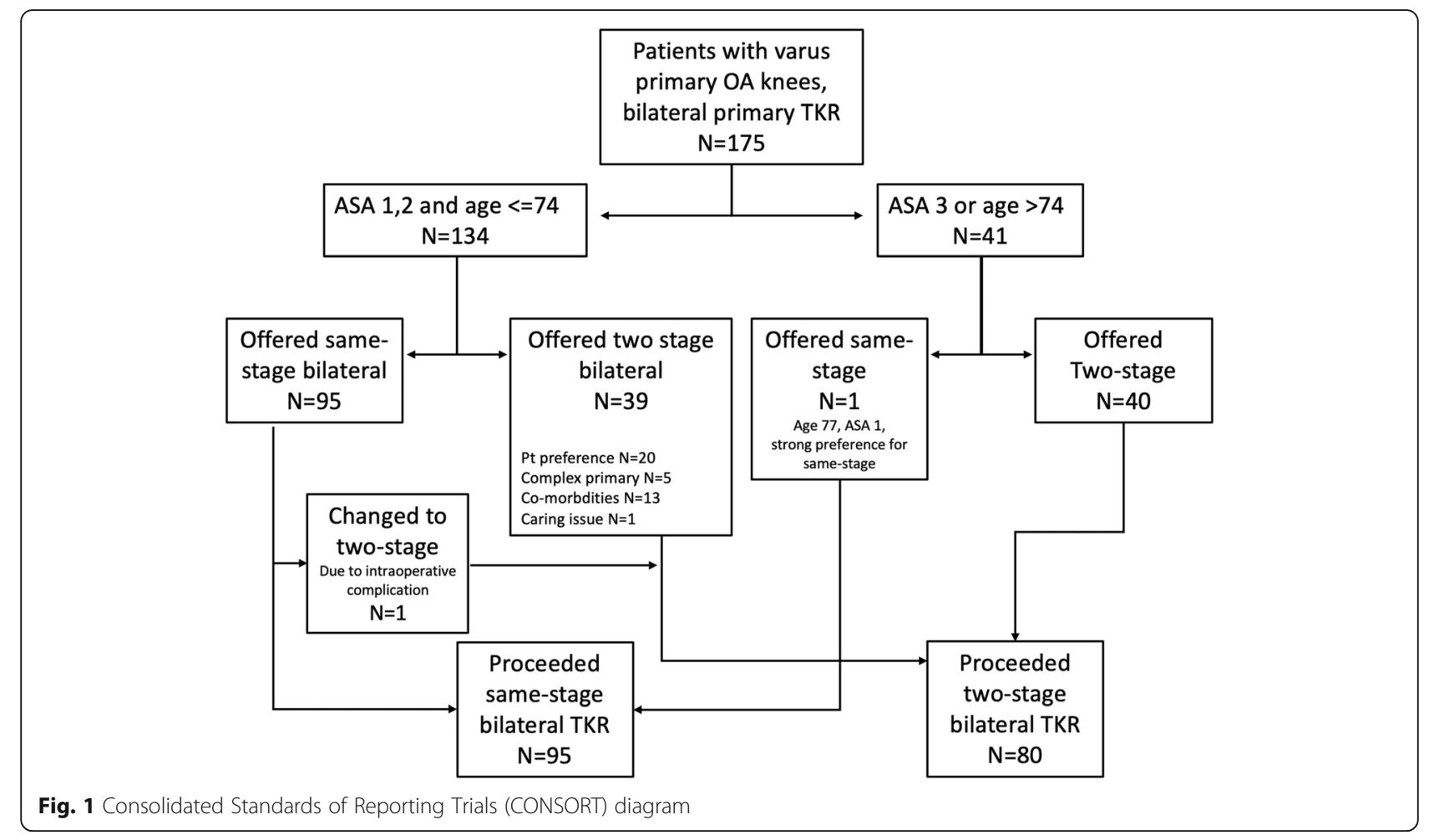

occupational therapists. All patients participated in a 4rweek 'pre-hab' programme, organised by physiotherapists and occupational therapists. An appropriate anaesthetic plan and a post-operative analgesic regimen were tailor-made. Any medical comorbidity requiring workup and optimisation was settled during a pre-operative in-patient medical consultation. Almost all TKR patients were admitted on the same day of surgery, except those requiring bridging therapy for warfarin and those requiring pace-maker adjustments. Iron-deficiency anaemia with haemoglobin $<10.0 \mathrm{~g} / \mathrm{dL}$ was treated by orally administering iron supplementation. JRC specialty nurses liaised with different parties to ensure fluent discharge process.

\section{Intra-operatively}

All TKR surgeries were performed by the same team of three senior specialist joint surgeons (FCH, KKB, HYW). The procedures were performed using tourniquets unless the distal pulses were not palpable, via the medial para-patella approach, with femoral computer navigation, and tibial extra-medullary guide. A variety of commercially available implant brands were used depending on availability and surgeon preferences. All prostheses were fixed with cement and tourniquet pressure was released for haemostasis. All patients received a periarticular injection of a $100-\mathrm{ml}$ cocktail $(20 \mathrm{ml} 0.5 \%$ levo-

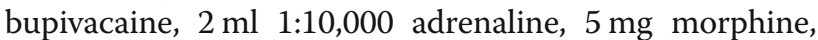
$30 \mathrm{mg}$ ketorolac (in $1 \mathrm{ml}$ ) and normal saline up to 100 $\mathrm{ml}$ ) before wound closure unless contraindicated (e.g. allergy or anaesthetically unfit). An intra-articular injection of $2 \mathrm{~g}$ of tranexamic acid was given. One suction drain was inserted only if the patient was taking double antiplatelet therapy or anticoagulants. Therefore, drains are always inserted in both knees if needed. In the case of same-stage, bilateral TKR, while one surgeon started closing the capsule of the first knee, another surgeon would start the incision on the other knee. Only one tourniquet was inflated at any time. Implant choice is decided by surgeons' preference and implant availability. It is our practice to always uses same implant for both knees to prevent patients' worry that one implant is better than the other.

\section{Post-operation}

All patients received multimodal orally administered analgesia. Pregabalin was started the day before TKR for 3 days. Paracetamol and etoricoxib were administered at post-operative day zero for 5 days. A weak orally administered opioid analgesic (tramadol) was administered for 3 days; it would be terminated early if patients complained of nausea and dizziness. Patient-controlled analgesia was prescribed by anaesthetists only when necessary and was typically limited to the first postoperative day.

Physiotherapists provided post-operative day-zero rehabilitation if the patient could tolerate it. In most cases, rehabilitation exercises were performed twice a day. 
Orally administered aspirin (80 mg daily for 2 weeks) was administered for thromboembolism prophylaxis unless contraindicated. Blood transfusion was protocoldriven. For a haemoglobin level $<10 \mathrm{~g} / \mathrm{dL}$, orally administered iron supplement was given. Patients were transfused only when haemoglobin was $<8 \mathrm{~g} / \mathrm{dL}$ or when they were symptomatic (including shortness of breath, tachycardia, palpitation, chest pain).

\section{Discharge and after discharge}

Patient discharge was also protocol-driven. They were allowed to return home when physiotherapists documented stable, independent frame walking and when pain control was reasonable; this usually occurred at post-operative days 3 to 4 .

A secure safety net that comprises JRC specialty nurses, physiotherapists, and occupational therapists was established during patient recovery at home. Outpatient physiotherapy was guaranteed on the third working day after discharge. Assistance from the JRC nursing clinic and from the JRC ward is available 24-hourly, and patients will not need to resort to emergency department attendance.

\section{Measurement of outcomes}

The data sources included the JRC Registry in an Excel file continuously updated by JRC specialty nurses, the Clinical Management System (the universal electronic health record system in Hong Kong) utilised by the Hospital Authority, and the rehabilitation database maintained by physiotherapists and occupational therapists.

Demographic data, including age, gender, ASA grade, pre-operative Knee Society Knee Score (KS), Knee Society Function Score (FS), and Oxford Knee Score (OKS), was captured. The KS, FS, and OKS were obtained by pre-operative occupation therapy assessment. ASA grades were retrieved by pre-operation anaesthetist assessment. Age, gender, implant choices, and drain usage were obtained from patient records.

Cost-effectiveness data included average length of stay (ALOS), operating theatre time (OTT), number of postoperative physiotherapy sessions (PhyS), and number of occupational therapy sessions (OccS) was captured. ALOS was obtained from patient records. Operation theatre turnover time was obtained from the operation theatre's record (automatically generated from anaesthetist's electronic record) of last operation's completion time (defined as completion of wound closure) to the next operation's anaesthesia-ready time (defined as completion of spinal anaesthesia injection, or completion of intubation in general anaesthesia, and ready for surgeons to position the patient) was obtained. The operation theatre turnover time is defined as the interval between last operation's completion time and the next operation's anaesthesia ready time. We sampled 4 months, from July to September 2017, and obtained the mean turnover time from the operation theatre's record. The cost of each day of hospital stay, physical therapy sessions and occupational therapy sessions is estimated by the gazetted public hospital charges on all non-entitled person (not covered by public welfare.

Operating theatre consumables include those used outside theatres (e.g. sterilisation and sterile packaging of re-usable instruments, autoclaves, etc.) and inside the theatre (e.g. personal protective gear, gloves, single-use drapes, etc.). The consumable cost is obtained from internal financial report for fiscal year 2017. The implant purchase cost per knee was equal for both groups.

Safety profile data included pre-operative haemoglobin (PreHb), haemoglobin level on the first day post-operation (HbD1), haemoglobin level drop between pre-operation and day 1 post-operation (HbDrop), transfusion percentage, transfusion volume (TV), surgical complication rate (SurgCR), medical complication rate (MedCR), and 30day and 90-day unplanned readmission rate (UPR).

The physiotherapist assessed patients using standard and commonly used tools from the Fullerton Functional Fitness Test Battery including the Thirty-second Chair Stand Test (CST) [13], the Timed Up and Go Test (TUGT) [12], and the Numeric Pain Rating Scale (NPRS). The CST requires the patient to sit upright that the patient sit in the middle of the chair, with crossed arms on the chest, keeps their feet flat and with a back straight. Then, upon the instruction word 'Go', the patient will rise to a straight standing position, then sit down to the starting position. The number of cycles that the patient can fully complete in $30 \mathrm{~s}$ is recorded; the more the better. The TUGT requires the patient to fully sit, stand up according to the therapist's command, walk for $3 \mathrm{~m}$, turn around, walk back to the chair and sit down. Walking aids can be used if needed. The time needed to complete the entire cycle is recorded; the lower this is the better the performance is. The NPRS involves asking the patient to rate their subjective pain score from 0 (no pain) to 10 (most severe pain).

Rehabilitation results were collected at different preoperative and post-operative times as follows:

- 'Pre-operation': when patients finished 'pre-hab'

- 'D0 post-op': when patients performed the first rehabilitation after the operation, typically on the same day post-operation if possible

- 'D/C from PT': when patients finished their final rehabilitation session and were discharged from outpatient physiotherapy.

\section{Data analysis}

A two-tailed Student's $T$ test was used for comparing the two groups' age, pre-operation KS, FS, OKS, ALOS, 
OTT, PhyS, OccS, PreHb, HbD1, HbDrop. $P<0.05$ is considered statistically significant.

The chi-square test was used in comparing ASA grade, gender, implant choice, drain use, transfusion percentage, transfusion volume (TV), SurgCR, MedCR, and 30day and 90-day UPR.

One-way analysis of variance (ANOVA) was used for CST, TUGT, and NPRS. Post-hoc subgroup analysis using a Bonferroni test was then used to compare the differences between the same-stage and first knee in the two-stage, between the same-stage and second knee in the two-stage, and between the first and second knee in the two-stage. In these analyses, $P<0.017$ was statistically significant (Bonferroni corrected $P$ value).

A 'young and fit' subgroup of two-stage patients that are similar to the same-stage group, namely non-obese, non-complex, age $<75$ years, and ASA grade 1 or 2 , was compared to the same-stage group to alleviate patient selection bias. The parameters that were compared included gender and number of patients, age, KS, FS, OKS, ALOS, transfusion percentage, $\mathrm{TV}$, reoperation rate (RR), SurgCR, and MedCR.

SPSS 21.0 (IBM Corp., Armonk, NY, USA) was used for statistical analysis.

\section{Results}

\section{Demographics}

Their demographics and pre-operation function scores are listed in Table 1 . The same-stage group had younger patients (65.6 (51-77, SD 5.31) vs 68.7 (56-79, SD 6.46), $P=0.041$ ) with a 'better' ASA composition (same-stage ASA $15.26 \%$, ASA $294.74 \%$, ASA $30 \%$, two-stage ASA 1 1.96\%, ASA 2 67.97\%, ASA 3 30.07\%; $P<0.001)$. The same-stage group had similar FS and OKS (Table 1). For $\mathrm{KS}$, the difference of mean value is small (same-stage 44.3 (18-79, SD 13.54, 95\% confidence interval (CI) 41.6-47) vs two-stage 44.5 (12-77, SD 12.82, 95\% CI $41.7-47.3)$, and is statistically insignificant as $95 \% \mathrm{CI}$ overlap.

The same-stage TKR and a subgroup of two-stage TKR patients ('young and fit' subgroup) with ASA grades $1-2$, age $<75$ years, non-complex, and non-obese, similar to the same-stage group, showed no significant difference in gender composition and age in two groups. The two groups have no difference in FS, KS, and OKS (Table 2).

Tourniquets were used for all cases. Drains were inserted in four knees (from two patients) from the twostage group and none in the same-stage group. No drains were used in the two-stage 'young and fit' subgroup. There was no statistically significant difference $(P=0.062)$. The implants used were all posterior stablising, including the Triathlon Knee System (Stryker, Kalamazoo, MI, USA) and the Attunes Knee System (DePuy
Synthes, West Chester, PA, USA) (Table 1). There was no significant difference in implant choices between two groups.

\section{Cost comparison}

The key information regarding cost comparison is listed in Table 1.

During the study period, Hong Kong public hospitals charged each non-entitled person HK\$4680 per day of inpatient stays and $\mathrm{HK} \$ 1110$ per specialist clinic visit, occupational therapy, and physiotherapy clinic session, which were assumed to be the unit cost. According to our centre's Sterile Supply Unit data for the fiscal year 2017, HK\$2421 (US\$312.3) was saved per same-stage, bilateral TKR procedure.

For each same-stage, bilateral TKR case performed instead of two-stage, bilateral TKR, the following resources were spared:

- 5.71 days of ALOS (estimated saving HK\$26,722.8)

- 3.34 (or 18.6\%) physiotherapy sessions (estimated saving $\mathrm{HK} \$ 11,733)$

- 3.78 (or 51.5\%) occupational therapy sessions (estimated saving HK\$4418)

- One set of operating theatre consumables (HK\$2421)

- 57.9 min (34-149) of operation theatre turnover time

- 27.4 min of operation theatre time

By considering these figures, we estimated that a total of HK $\$ 45,295$ or US $\$ 5822$ (US $\$ 1=$ HK\$7.78) could be saved for every patient who underwent same-stage, bilateral TKR rather than two-stage, bilateral TKR. This estimated a cost saving of $27.6 \%$ per TKR.

When comparing same-stage group with the two-stage 'young and fit' subgroup, a 4.65 days' shorter average length of stay (ALOS) was saved (Table 2).

\section{Safety profile}

The same-stage group initially had a slightly better blood haemoglobin level (same-stage $13.50 \mathrm{~g} / \mathrm{dL},(9.8-16.6, \mathrm{SD}$ $1.10,95 \%$ CI 13.3-13.7) vs two-stage $13.00 \mathrm{~g} / \mathrm{dL}(9.1-$ 15.7, SD 1.31, 95\% CI 12.7-13.3); $P=0.002$ ) but experienced a haemoglobin drop $0.96 \mathrm{~g} / \mathrm{dL}$ more than the twostage group (same-stage $3.03(0.0-6.0$, SD 1.21, 95\% CI $2.79-3.27)$ vs two-stage $2.07(-0.1-6.2$, SD $1.01,95 \% \mathrm{CI}$ $1.85-2.29) ; P<0.001)$. However, this difference did not translate into a higher transfusion volume (same-stage $0.18(0-6$, SD $0.45,95 \%$ CI $0.090-0.27)$ vs two-stage $0.09(0-4$, SD $0.76,95 \%$ CI $-0.52-0.70) ; P=0.116)$ or a higher transfusion rate (same-stage $8.42 \%$ (16 of 190 knees) vs two-stage $3.75 \%$ (6 of 160 knees); $P=0.078$ ). There were no statistically significant differences in 
Table 1 Results of comparison between same-stage and two-stage total knee replacements (TKRs)

\begin{tabular}{|c|c|c|c|}
\hline Parameters & Same-stage, bilateral TKR $(n=95)$ & Two-stage, bilateral TKR $(n=80)$ & $P$ \\
\hline \multicolumn{4}{|l|}{ Demographics } \\
\hline Age (years) & $65.6(51-77$, SD 5.31) & $68.7(56-79$, SD 6.46) & 0.041 \\
\hline Gender & $26 \mathrm{M}, 69 \mathrm{~F}$ & $22 \mathrm{M}, 58 \mathrm{~F}$ & 0.894 \\
\hline \multirow[t]{3}{*}{ ASA grade } & 1: $5,5.26 \%$ & $1: 3,3.75 \%$ & \multirow[t]{3}{*}{$<0.001$} \\
\hline & $2: 90,94.74 \%$ & $2: 54,67.97 \%$ & \\
\hline & 3: $0 \%$ & $3: 24,30.07 \%$ & \\
\hline Implant choice & $\begin{array}{l}\text { Triathlon } 124(65.3 \%) \\
\text { Attune } 66(34.7 \%)\end{array}$ & $\begin{array}{l}\text { Triathlon } 112(70.0 \%) \\
\text { Attune } 48(30.0 \%)\end{array}$ & 0.505 \\
\hline \multicolumn{4}{|l|}{ Pre-operation function } \\
\hline \multirow[t]{2}{*}{ FS } & $50.2(5-80$, SD 13.19, & 47.3 (5-90, SD 16.00, & \multirow[t]{2}{*}{0.706} \\
\hline & $95 \%$ Cl 47.7-52.9) & $95 \%$ Cl 43.8-50.8) & \\
\hline \multirow[t]{2}{*}{ KS } & 44.3 (18-79, SD 13.54, & 44.5 (12-77, SD 12.82, & \multirow[t]{2}{*}{0.025} \\
\hline & $95 \% \mathrm{Cl} 41.6-47)$ & $95 \%$ Cl 41.7-47.3) & \\
\hline \multirow[t]{2}{*}{ OKS } & $24.9(8-54$, SD 6.69 & $25.2(6-54$, SD 7.62 & \multirow[t]{2}{*}{0.855} \\
\hline & $95 \%$ Cl 23.5-26.3) & $95 \%$ Cl 23.5-26.9) & \\
\hline \multicolumn{4}{|l|}{ Cost-effectiveness } \\
\hline \multirow[t]{3}{*}{ LOS (days) } & $6.64(3-12$, SD 2.14, & $12.35^{\mathrm{a}}(7-65, \mathrm{SD} 6.67$ & \multirow[t]{2}{*}{$<0.001$} \\
\hline & $95 \%$ Cl 6.21-7.07) & $95 \%$ Cl 10.9-13.8) & \\
\hline & & $6.07^{\mathrm{b}}(2-43)$ & $<0.001$ \\
\hline \multirow[t]{2}{*}{$\mathrm{OTT}^{\mathrm{c}}$ (mins) for 2 knees } & $152.8^{\mathrm{C}}(68-478$, SD 31.34 & $180.3^{c}\left(111-417^{c}\right.$, SD 48.46 & \multirow[t]{2}{*}{$<0.001$} \\
\hline & $95 \% \mathrm{Cl} 147.1-159.2)$ & $95 \%$ Cl 170-191) & \\
\hline \multirow[t]{2}{*}{ Phys } & $14.56^{\mathrm{d}}(3-26, \mathrm{SD} 4.78$ & $17.90^{d}(11-37$, SD 4.68 & \multirow[t]{2}{*}{$<0.001$} \\
\hline & $95 \%$ Cl 13.5-15.6) & $95 \%$ Cl 17.0-18.8) & \\
\hline \multirow[t]{2}{*}{ Occs } & $3.56^{\mathrm{d}}(2-8, \mathrm{SD} 1.97$ & $7.34^{\mathrm{d}}(2-24, \mathrm{SD} 2.40$ & \multirow[t]{2}{*}{$<0.001$} \\
\hline & $95 \%$ Cl 3.16-3.96) & $95 \%$ Cl 6.81-7.87) & \\
\hline \multicolumn{4}{|l|}{ Safety } \\
\hline \multirow[t]{2}{*}{ PreHb (g/dL) } & 13.50 (9.8-16.6, SD 1.10, & $13.00(9.1-15.7$, SD 1.31, & \multirow[t]{2}{*}{0.002} \\
\hline & $95 \%$ Cl 13.3-13.7) & $95 \%$ Cl 12.7-13.3) & \\
\hline \multirow[t]{2}{*}{$\mathrm{HbD} 1(\mathrm{~g} / \mathrm{dL})$} & 10.47 (7.0-14.1, SD 1.28, & 10.93 (7.4-14.1, SD 1.43, & \multirow[t]{2}{*}{0.003} \\
\hline & $95 \%$ Cl 10.2-10.7) & $95 \%$ Cl 10.6-11.2) & \\
\hline \multirow[t]{2}{*}{ HbDrop (g/dL) } & $3.03(0.0-6.0$, SD 1.21, & $2.07(-0.1-6.2$, SD 1.01, & \multirow[t]{2}{*}{$<0.001$} \\
\hline & $95 \%$ Cl 2.79-3.27) & $95 \%$ Cl 1.85-2.29) & \\
\hline Transfusion percentage & $8.42 \%$ ( 16 of 190 knees) & $3.75 \%$ (6 of 160 knees) & 0.078 \\
\hline \multirow[t]{2}{*}{ TV (units) for 2 knees } & $0.18(0-6$, SD 0.45, & $0.09(0-4$, SD 0.76, & 0.116 \\
\hline & $95 \%$ Cl 0.090-0.27) & $95 \% \mathrm{Cl}-0.52-0.70)$ & \\
\hline SurgCR & 7.37\% (7 of 95 operations) & $5.63 \%$ (9 of 160 operations) & 0.511 \\
\hline MedCR & $3.68 \%$ (7 of 190 knees) & $5.63 \%$ (9 of 160 knees) & 0.386 \\
\hline & $10.5 \%$ (10 of 95 operations) & $6.20 \%$ (10 of 160 operations) & 0.155 \\
\hline $\mathrm{RR}$ & $3.16 \%$ (3 of 95 operation) & $4.38 \%$ (7 of 160 operations) & 0.548 \\
\hline 30-days UPR & $2.11 \%$ (2 of 95 operations) & $6.92 \%$ (11 of 160 operations) & 0.094 \\
\hline 90-days UPR & $4.21 \%$ (4 of 95 operations) & $9.43 \%$ (15 of 160 operations) & 0.129 \\
\hline
\end{tabular}

Abbreviations: ALOS average length of stay, ASA American Society of Anesthesiologists, FS Knee Society Function Score, HbD1 haemoglobin level on first day postoperation, HbDrop haemoglobin level drop, KS Knee Society Knee Score, MedCR medical complication rate, OKS Oxford Knee Score, OccS occupational therapy sessions, OTT operation theatre time, PhyS physical therapy sessions, PreHb pre-operative haemoglobin, RR reoperation rate, $S D$ standard deviation, SurgCR surgical complication rate, $T V$ transfusion volume, UPR unplanned readmission, $95 \% \mathrm{Cl} 95 \%$ confidence interval

Statistically significant $P$ values are presented in bold

${ }^{a}$ patient-specific total ALOS of 2 knees in an individual patient

${ }^{b}$ knee-specific ALOS of one knee

cpresented as the total operation time for both knees

dpresented as the total number for both knees 
Table 2 Results of comparison between same-stage and two-stage 'young and fit' subgroup (non-obese, non-complex, ASA 1 or 2, age $<75$ years)

\begin{tabular}{|c|c|c|c|}
\hline Subgroup analysis demographics & Same-stage, bilateral TKR $(n=95)$ & $\begin{array}{l}\text { Two-stage, bilateral TKR 'young and fit' } \\
\text { subgroup }(n=22)\end{array}$ & $P$ \\
\hline Gender and number & $26 \mathrm{M}, 69 \mathrm{~F}$ & $6 \mathrm{M}, 16 \mathrm{~F}$ & 0.556 \\
\hline Age & $65.6(51-77$, SD 5.31, 95\% Cl 64.5-66.7) & $66.1(56-74$, SD 4.94, 95\% Cl 64-68.2) & 0.415 \\
\hline \multirow[t]{3}{*}{ ASA grade } & 1: 5, 5.26\%, & $1: 3,13.64 \%$ & 0.161 \\
\hline & $2: 90,94.74 \%$ & 2: $19,86.36 \%$ & \\
\hline & $3: 0,0 \%$ & $3: 0,0 \%$ & \\
\hline \multirow[t]{2}{*}{ FS } & $50.3(5-80$, SD 12.99, & 50.1 (5-90, SD 18.64, & 0.083 \\
\hline & $95 \%$ Cl 47.7-52.9) & $95 \%$ Cl 42.3-57.9) & \\
\hline \multirow[t]{2}{*}{ KS } & $44.3(18-79$, SD 13.54, & $44.7(12-74$, SD 12.97, & 0.412 \\
\hline & $95 \%$ Cl 41.6-47) & $95 \%$ Cl 39.3-50.1) & \\
\hline \multirow[t]{2}{*}{ OKS } & $24.9(8-54$, SD 6.69 & $25.3(6-54$, SD 8.41, & 0.556 \\
\hline & $95 \%$ Cl 23.5-26.3) & $95 \%$ Cl 21.5-28.5) & \\
\hline \multirow[t]{2}{*}{ ALOS (Days) } & $6.64(3-12$, SD 2.13, & $11.29^{\mathrm{a}}(7-15$, SD 1.55 & $<0.001$ \\
\hline & $95 \% \mathrm{Cl} 6.21-7.07)$ & $95 \%$ Cl 10.6-11.9) & \\
\hline \multirow[t]{2}{*}{ HbDrop (g/dL) } & $3.03(0.0-6.0$, SD 1.21, & $2.20(-0.1-6.2$, SD 1.02, & $<0.001$ \\
\hline & $95 \%$ Cl 2.19-3.27) & $95 \%$ Cl 1.77-2.63) & \\
\hline \multirow[t]{2}{*}{ TV (units) for 2 knees } & $0.18(0-6$, SD 1.21, & $0.10(0-4$, SD 1.02, & 0.289 \\
\hline & $95 \% \mathrm{Cl}-0.063-0.42)$ & $95 \% \mathrm{Cl}-0.36-0.53)$ & \\
\hline Transfusion percentage & $8.42 \%$ (16 of 190 knees) & $9.09 \%$ (4 of 44 knees) & 0.886 \\
\hline $\mathrm{RR}$ & $3.16 \%$ (6 of 190 knees) & $6.81 \%$ (3 of 44 knees) & 0.255 \\
\hline Surg CR & $3.68 \%$ (7 of 190 knees) & $9.09 \%$ (4 in 44 knees) & 0.127 \\
\hline Med CR & $10.5 \%$ (10 of 95 operations) & $4.55 \%$ (2 of 44 operations) & 0.243 \\
\hline
\end{tabular}

Abbreviations: ALOS average length of stay, ASA American Society of Anesthesiologists, FS Knee Society Function Score, HbD1 haemoglobin level on first day postoperation, HbDrop haemoglobin level drop, KS Knee Society Knee Score, MedCR medical complication rate, OKS Oxford Knee Score, OccS occupational therapy sessions, OTT operation theatre time, PhyS physical therapy sessions, PreHb pre-operative haemoglobin, $R R$ reoperation rate, $S D$ standard deviation, SurgCR surgical complication rate, $T V$ transfusion volume, UPR unplanned readmission, 95\% Cl 95\% confidence interval Statistically significant $P$ values are presented in bold

a patient-specific total ALOS of 2 knees in an individual patient

${ }^{b}$ knee-specific ALOS of one knee

cpresented as the total operation time for both knees

${ }^{d}$ presented as the total number for both knees

SurgCR, MedCR, RR, 30-day UPR or 90-day UPR (Table 1 ). HbD1 has no statistically significant difference as 95\% CIs overlap (same-stage 10.47 (7.0-14.1, SD 1.28, 95\% CI 10.2-10.7) vs two-stage 10.93 (7.4-14.1, SD 1.43, 95\% CI 10.6-11.2)]. All transfusion occurred with $\mathrm{Hb}<$ $8.0 \mathrm{~g} / \mathrm{dL}$.

The causes of reoperation and surgical and medical complications are listed in Table 3. There were no cardiovascular events, pulmonary embolism or mortality in either group.

We also compared the same-stage TKR and a subgroup of two-stage TKR patients ('young and fit' subgroup) with ASA $1-2$, age $<75$ years, non-complex, and non-obese in the two-stage group. The results are listed in Tables 2 and 4. HbDrop was $0.83 \mathrm{~g} / \mathrm{dL}$ more in the same-stage group (same-stage 3.03 (0.06.0, SD 1.21, 95\% CI 2.19-3.27) vs two-stage 2.20 (0.1-6.2, SD 1.02, 95\% CI 1.77-2.63); $P<0.001)$, but this is statistically insignificant as $95 \%$ CIs overlap. There was no statistically significant difference in transfusion volume (TV), transfusion percentage, RR, SurgCR or MedCR (Table 2).

\section{Rehabilitation}

The rehabilitation results are listed in Fig. 2 and Table 5 and demonstrate rehabilitation outcomes at different times between the same-stage and two-stage, bilateral TKR.

Before operation, the same-stage group performed similarly in the TUGT and slightly better in the CST (same-stage 11.64 (SD 4.72) vs two-stage first knee 10.03 (SD 3.20), two-stage second knee 11.28 (SD 2.79), $P=$ 0.006). The same-stage group experienced a higher pain level (NPRS) before surgery (same-stage 7.69 (SD 1.48), two-stage first knee 6.08 (SD 1.85), two-stage second knee 6.34 (SD 2.15), $P<0.001$ ). 
Table 3 Reoperation causes, surgical and medical complications

\begin{tabular}{|c|c|c|}
\hline Complications and reoperation causes & Same-stage, bilateral TKR & Two-stage, bilateral TKR \\
\hline \multicolumn{3}{|l|}{ Reoperation causes } \\
\hline Persistent wound discharge requiring irrigation and debridement & 2 & 4 \\
\hline Wound haematoma requiring open drainage & 1 & 1 \\
\hline Periprosthetic infection & & 1 \\
\hline Intra-articular haematoma & & 1 \\
\hline \multicolumn{3}{|l|}{ Surgical complications } \\
\hline Reoperation causes above-mentioned & 3 & 7 \\
\hline Medial collateral ligament injury/avulsion & 2 & \\
\hline Patella-tendon insertion avulsion & 1 & \\
\hline Intra-operative fracture & 1 & 2 \\
\hline Popliteal artery laceration & & 1 \\
\hline \multicolumn{3}{|l|}{ Medical complications } \\
\hline Ischaemic stroke & 1 & \\
\hline Chest infection & 4 & \\
\hline Urinary tract infection & 2 & 4 \\
\hline Acute renal failure & & 1 \\
\hline Insomnia & 1 & \\
\hline Gastrointestinal bleeding & 1 & \\
\hline Deep vein thrombosis & 1 & \\
\hline Congestive heart failure & & 1 \\
\hline Aspirin-induced gastrointestinal upset & & 1 \\
\hline Transient fast atrial fibrillation & & 1 \\
\hline Transient hypotension (resolved with only fluid replacement) & & 1 \\
\hline Common peroneal nerve palsy & & 1 \\
\hline
\end{tabular}

TKR total knee replacement

During the first rehabilitation post-operation, samestage patients performed worse in the Thirty-second Chair Stand Test (same-stage 0.48 (SD 1.52) vs twostage first knee 3.69 (SD 3.71), two-stage second knee 4.69 (SD 4.00), $P=<0.001$ ), and worse in the TUGT (same-stage 48.45 (SD 17.48) vs two-stage first knee 44.14 (SD 21.23), two-stage second knee 37.02 (SD $24.45), P=0.005)$. However, the for pain level there is no difference for the two-stage procedure regardless of whether for the first or second knee [same-stage 4.74 (SD 1.60) vs two-stage first knee 4.46 (SD 2.07), twostage second knee 5.00 (SD 1.88), $P=2.61$ ).

At the final rehabilitation post-operation, same-stage patients performed better than two-stage patients in the TUGT (same-stage 10.57 (SD 2.78) vs two-stage first knee 14.91 (SD 5.19), two-stage second knee 11.73 (SD

Table 4 Reoperation causes, medical and surgical complications of two-stage, bilateral TKR 'young and fit 'subgroup with ASA 1 or 2 , age $<75$ years, non-complex, non-obese

\begin{tabular}{ll}
\hline Complications and reoperation causes & Two-stage, bilateral TKR 'young and fit' subgroup \\
\hline Reoperation causes & 3 \\
Persistent wound discharge requiring irrigation and debridement & 3 \\
Surgical complications & 1 \\
Reoperation causes above-mentioned & \\
Popliteal artery laceration & 1 \\
Medical complications & 1 \\
Transient fast atrial fibrillation & 1 \\
Urinary tract infection & \\
\hline
\end{tabular}




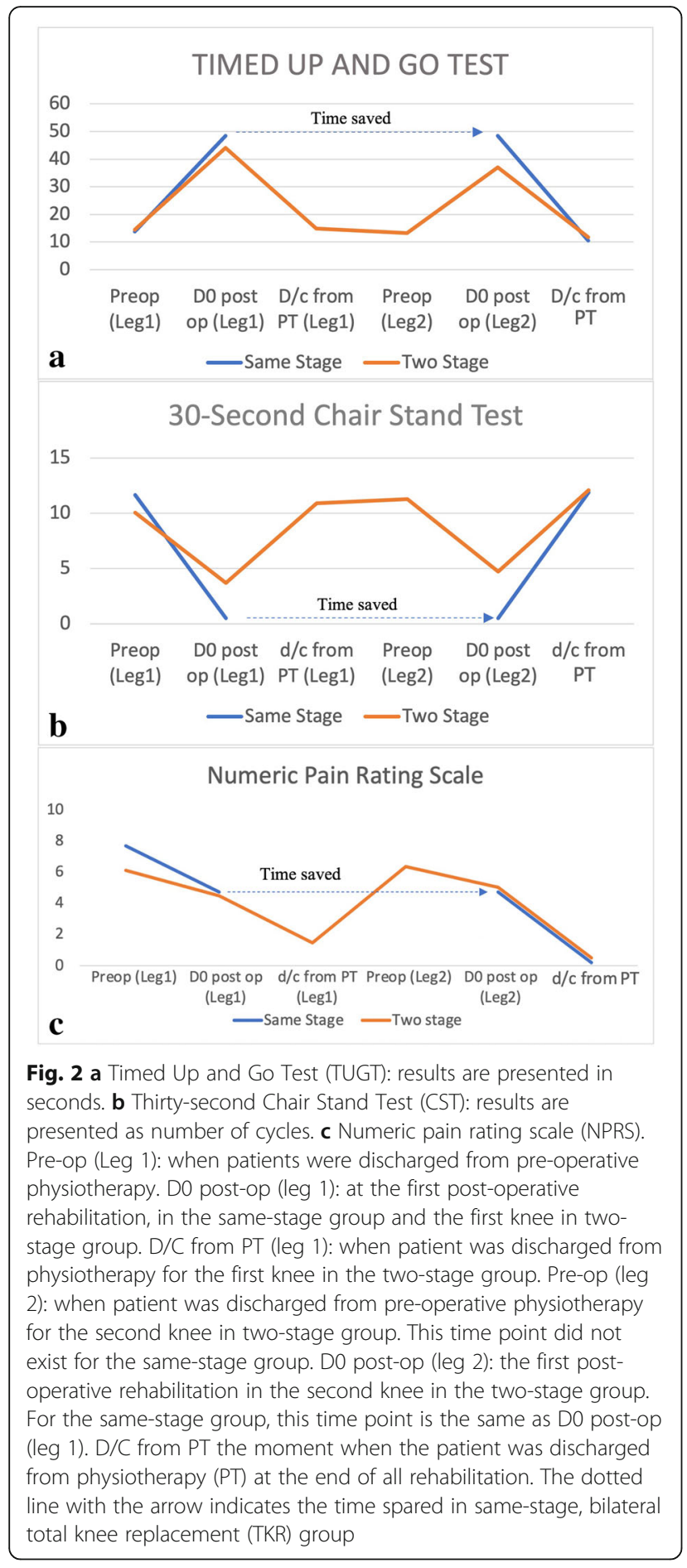

3.02), $P=0.003)$, they performed with no difference in the Thirty-second Chair Stand Test (same-stage 11.86 (SD 3.27) vs two-stage first knee 10.88 (SD 3.06), twostage second knee 12.08 (SD 2.96), $P=0.067$ ), and reported lower pain levels (same-stage 0.19 (SD 0.83) vs two-stage first knee 1.44 (SD 1.51), two-stage second knee 0.48 (SD 0.97), $P<0.001$ ).
Comparison is made among two-stage patients for the first and second knee by post-hoc analysis. At the end of rehabilitation, the second knee performed better than the first knee in the TUGT (14.91 (SD 5.19) vs 11.73 (SD 3.02), $P<0.001$ ) but no difference in the Thirtysecond Chair Stand Test (10.88 (SD 3.06) vs 12.08 (SD 2.96), $P=0.067$ ). The pain level has improved (first knee 1.44 (SD 1.51) vs second knee 0.48 (SD 0.97), $P<0.001$ ).

\section{Discussion}

We have demonstrated that same-stage, bilateral TKR can reduce cost without significant compromise on safety profile and rehabilitation performance. There was no significant difference in surgical complication rate, medical complication rate, readmission rate, and 30-day and 90-day unplanned readmission rate. The haemoglobin drop difference is narrow although statistically significant (less than $1 \mathrm{~g} / \mathrm{dL}$ ), and unlikely clinically significant with no difference in transfusion volume or transfusion rate. The safety profile comparison is not confounded by same-stage group's better ASA grading, younger age, non-complexity, and non-obesity, according to a subgroup analysis of the 'young and fit' subgroup. The haemoglobin drop difference is statistically insignificant as $95 \%$ CIs overlap, and the difference in mean value is narrow $(0.83 \mathrm{~g} / \mathrm{dL})$. There is no difference in transfusion rate or transfusion volume (Table 2).

Our results of about $27 \%$ saving of cost are comparable to those of Reuben et al., who reported a 36\% reduction in cost for same-stage compared to two-stage TKR procedures [14]. For each same-stage, bilateral TKR patient, a total of $85.3 \mathrm{~min}$ could be spared (operation theatre time of $27.4 \mathrm{~min}$ and turnover time of 57.9 min). Assuming a theatre operates from 8 am to $5 \mathrm{pm}$ (9 h) for elective cases, this means a $15.8 \%$ increase in efficiency, or one more case can be performed for every 6.33 cases. This factor is not quantifiable in terms of money. The patient load is large, and even small efforts to improve cost-effectiveness will make a huge cumulative difference.

Conflicting reports on the cost-effectiveness of samestage versus two-stage, bilateral TKR can be explained by different settings, practices, and remuneration algorithms, among other factors [13]. Recently, Philips et al. reported that same-stage, bilateral TKR had lower perepisode inpatient cost but the same 90-day episodespecific cost as two-stage, bilateral TKR [5]. The reported cause was a higher outpatient rehabilitation facility cost, which neutralised the shorter inpatient rehabilitation. This study is based in a North America setting. In Hong Kong there is no outpatient rehabilitation facilities and patients are discharged when fit to stay at home. The unplanned readmission rate was noninferior in our same-stage, bilateral TKR patients. Our 
Table 5 Details for the (a) Timed Up and Go Test (TUGT) (b) 30-s Chair Stand Test (CST), (c) Numeric Pain Rating Scale (NPRS)

\begin{tabular}{|c|c|c|c|}
\hline TUGT & Pre-operation (in seconds) & $\begin{array}{l}\text { D0 post-op } \\
\text { (in seconds) }\end{array}$ & $\begin{array}{l}\mathrm{D} / \mathrm{C} \text { from } \mathrm{PT} \\
\text { (in seconds) }\end{array}$ \\
\hline Same-stage & 13.78 (SD 8.09) & 48.45 (SD 17.48) & 10.57 (SD 2.78) \\
\hline Two-stage 1st knee & 14.40 (SD 5.77) & 44.14 (SD 21.23) & 14.91 (SD 5.19) \\
\hline Two-stage 2nd knee & 13.28 (SD 6.10) & 37.02 (SD 24.45) & 11.73 (SD 3.02) \\
\hline ANOVA $^{a} P$ value & 0.112 & 0.005 & 0.003 \\
\hline \multicolumn{4}{|l|}{ Post-hoc Bonferroni test ${ }^{\mathrm{b}}$} \\
\hline Same-stage vs 1st knee & - & 0.437 & $<0.001$ \\
\hline Same-stage vs 2 nd knee & - & 0.003 & 0.003 \\
\hline 1st knee vs 2nd knee & & 0.141 & $<0.001$ \\
\hline CST & $\begin{array}{l}\text { Pre-operation } \\
\text { (no. of cycles) }\end{array}$ & $\begin{array}{l}\text { D0 post-op } \\
\text { (no. of cycles) }\end{array}$ & $\begin{array}{l}\text { D/C from PT } \\
\text { (no. of cycles) }\end{array}$ \\
\hline Same-stage & 11.64 (SD 4.72) & 0.48 (SD 1.52) & 11.86 (SD 3.27) \\
\hline Two-stage 1st knee & 10.03 (SD 3.20) & 3.69 (SD 3.71) & 10.88 (SD 3.06) \\
\hline Two-stage 2nd knee & 11.28 (SD 2.79) & 4.69 (SD 4.00) & 12.08 (SD 2.96) \\
\hline ANOVA $^{a} P$ value & 0.006 & $<0.001$ & 0.067 \\
\hline \multicolumn{4}{|l|}{ Post-hoc Bonferroni test ${ }^{b}$} \\
\hline Same-stage vs 1st knee & 0.001 & $<0.001$ & - \\
\hline Same-stage vs 2nd knee & 0.344 & 0.001 & - \\
\hline 1st knee vs 2nd knee & 0.297 & 0.289 & - \\
\hline NPRS & $\begin{array}{l}\text { Pre-operation } \\
\text { (score 0-10) }\end{array}$ & $\begin{array}{l}\text { D0 post-op } \\
\text { (score } 0-10)\end{array}$ & $\begin{array}{l}\text { D/C from PT } \\
\text { (score } 0-10)\end{array}$ \\
\hline Same-stage & 7.69 (SD 1.48) & 4.74 (SD 1.60) & 0.19 (SD 0.83) \\
\hline Two-stage 1st knee & 6.08 (SD 1.85) & 4.46 (SD 2.07) & 1.44 (SD 1.51) \\
\hline Two-stage 2nd knee & 6.34 (SD 2.15) & 5.00 (SD 1.88) & 0.48 (SD 0.97) \\
\hline ANOVA $^{a} P$ value & $<0.001$ & 0.261 & $<0.001$ \\
\hline \multicolumn{4}{|l|}{ Post-hoc Bonferroni test ${ }^{b}$} \\
\hline Same-stage vs 1st knee & $<0.001$ & - & 0.001 \\
\hline Same-stage vs 2nd knee & 0.001 & - & 0.003 \\
\hline 1st knee vs 2nd knee & 0.820 & - & $<0.001$ \\
\hline
\end{tabular}

Abbreviations: ANOVA analysis of variance, DO post-op the first post-operative rehabilitation results in the second knee in two-stage group, D/C from PT the moment when the patient was discharged from physiotherapy (PT) at the end of all rehabilitation

${ }^{a}$ One-way ANOVA

${ }^{\mathrm{b}}$ Post-hoc Bonferroni $P$ value correction: significant if $P<0.0170$

ERAS protocol contributes significantly to the shorter length of stay.

Besides cost, our safety profile for same-stage surgery was similar-or even better-compared to two-stage surgery in all parameters. Similarly, a systemic review by Malahias et al. [15] reviewed 19 articles that concurred with other studies $[16,17]$ that mortality, revision rate, complication rates, thromboembolic events, and cardiac complications were at least similar or even improved for same-stage compared to two-stage TKR patients.

Few studies that have compared same-stage versus two-stage, bilateral TKR have separately compared the two-stage, bilateral TKR younger and fitter patient subgroup. This endeavour resulted in selection bias because healthier patients were selected for same-stage, bilateral
TKRs in most cases, confounding the advantageous findings of same-stage, bilateral TKR. Our study alleviated this bias by separately comparing the two-stage, bilateral TKR subgroup with ASA 1 or 2 , age $<75$ years, nonobese, and non-complex. This subgroup comparison produced the same results as a the general comparison of the same-stage versus the two-stage TKR. This result is more convincing in demonstrating that same-stage, bilateral TKR is safe and not confounded by selection bias.

Compared to other studies, our same-stage, bilateral TKR group had a significantly lower transfusion percentage than other studies, including one recent study that reported rates of $15.8 \%$ for same-stage TKRs and $6.2 \%$ for two-stage TKRs [11]. This difference may suggest that our protocol, including refraining from drain 
insertion and iron-therapy for anaemia $>8 \mathrm{~g} / \mathrm{dL}$, is useful. Being conservative in terms of blood transfusion did not cause any cardiopulmonary incidents in our study.

Although increasing evidence has demonstrated the safety of same-stage, bilateral TKRs, including this study, current overriding opinions are still conservative in recommending same-stage, bilateral TKRs to patients [13], especially to patients with cardiopulmonary complications [18]. We believe that this study clearly defines a safe and well-proven perioperative pathway and patient selection criteria that other centres can follow and then reproduce our optimal results, so that appropriate patients are not excluded from same-stage, bilateral TKRs merely due to unnecessary safety concern.

Despite the same-stage operation having a noninferior safety profile, some patients refuse same-stage because of worry of pain and poor walking performance. In this study, $40.8 \%$ of patients fit the same-stage operation but chose a two-stage operation due to their subjective wish. Our study can further alleviate pain and rehabilitation concern.

Some patients worry about poor performance after both knee surgery types, and think that they would perform better and have less pain if the operating was performed one knee at a time. Some patients may think that pain after the first knee in a two-stage operation is better than same-stage operation. Our results showed the contrary. In fact in the literature, studies have compared final functional results after same-stage versus two-stage, bilateral TKRs. However, to our knowledge, few studies have compared each time point in detail, particularly using the results after the first knee replacement in a two-stage, bilateral TKR. This is the merit of our study. We showed that same-stage surgery has better performance compared to the two-stage first knee operation in the TUGT while there was no difference in the Thirty-second Chair Stand Test. Pain level is better in same-stage surgery than in two-stage first knee surgery. Same-stage surgery is at least non-inferior to twostage first knee in clinical outcomes.

By the end of rehabilitation, the same-stage group performed better in the TUGT and there was no difference in the Thirty-second Chair Stand Test. It showed that for same-stage surgery patients could enjoy a better, or at least non-inferior, functional outcome sooner than two-stage patients (sooner because only one surgery instead of two surgeries are required). This outcome is especially important in a locality where the waiting period for any knee replacement in the public healthcare system is long. A systemic review by Malahias et al. also revealed similar findings [15], that same-stage, bilateral TKR show improvement in some parameters and similar in others. This result could be due to the absence of painful knees that have not been operated on in same- stage patients, and that in two-stage, bilateral TKRs, the flexion contracture of the knee that has not been operated on will hinder the function of the operated knee [19]. Selection bias (selecting 'better' patients as in our study) may also underlie this finding.

For pain level, we found that the pain level in the same-stage group was similar to the two-stage group, immediately post-operation or when discharged from physiotherapy, even though the baseline pain level for the same-stage group was worse. This result is likely due to the multimodal analgesia administered intraoperatively and post-operatively according to our protocol. Similarly, Bagsby et al. reviewed 697 knees subjected to same-stage, bilateral TKR or unilateral TKR and found no difference in the pain level [20]. This finding may significantly affect patients' decision-making and encourage them to choose same-stage, bilateral TKRs, because some patients avoid same-stage surgery due to worry of worse pain.

An age threshold of 75 years was chosen. The Literature found an increased surgical risk and anaesthetic risk for patients age older than 75 years [21-23]. Other thresholds including 80 years [24] or 85 years [25] were studied, but the benefit may be limited by a patient's life expectancy. Thresholds of 70 were proposed [26], but this narrow indication may unnecessarily deprive suitable patients from the benefit of same-stage, bilateral TKR.

\section{Limitations}

This study only represents a single centre's experience with a limited sample size relative to other large registry data analyses. However, many outcomes, e.g. cost, transfusion percentage, thromboembolism prevalence, etc. depend heavily on perioperative practices, e.g. our centre's unique ERAS protocol. It is the outcome of the entire patient management pathway rather than a surgery strategy itself that is compared. The fact that this is a single-centre study ensured homogeneity in patient selection and perioperative routines, including our ERAS protocol, which avoided confounding factors in larger, multi-centre studies. Only two patients (2.5\%) from the two-stage group received drain insertion, and this number is unlikely to cause significant differences.

\section{Conclusion}

This study demonstrates that same-stage, bilateral TKR can reduce healthcare costs, with non-inferior safety profiles and rehabilitation outcomes. The prerequisite is a suitable patient selection and perioperative protocol. Same-stage, bilateral TKRs can be recommended to patients who fulfil the selection criteria and could benefit patients and the entire healthcare system. 


\section{Acknowledgements}

The authors thank Ms. Vikki Chu, Ms. SW Wong, and Ms. OY Wong and other staff from the Joint Replacement Centre of Department of Orthopaedics and Traumatology, Alice Ho Miu Ling Nethersole Hospital, for their help in this study. The authors also thank Prof. Kevin KW Ho (Department of Orthopaedics and Traumatology, Chinese University of Hong Kong) and Dr. Henry Pang (Department of Orthopaedics and Traumatology, Prince of Wales Hospital) for their contributions during writing of this manuscript.

\section{Authors' contributions}

The initial of the authors are: Raymond CW Wan $=$ RW, Jason CH Fan $=J F$, Yuk-Wah Hung $=\mathrm{YH}$, Ka-Bon Kwok $=\mathrm{KK}$, Carmen $\mathrm{KM} L \mathrm{LO}=\mathrm{CL}$, Kwong-Yin Chung $=$ KC. RW acquired data, performed statistical analysis, and prepared the manuscript. JF is the senior author who conceptualised the study, designed the study protocol, ensured study protocol adherence, and contributed equally to writing of the manuscript. YH and KK contributed significantly to study protocol design, ensured adherence to the study proto$\mathrm{col}$, and data acquisition. KL contributed significantly to the acquisition of data and performance of the study protocol. KC contributed to conceptualisation of study and manuscript writing. All authors read and approved the final manuscripts.

\section{Funding}

This research did not receive any specific grant from funding agencies in the public, commercial or not-for-profit sectors.

\section{Availability of data and materials}

The dataset analysed during the current study is available from the corresponding author on reasonable request.

\section{Declarations}

\section{Ethics approval and consent to participate}

Ethics approval was obtained from the Chinese University of Hong Kong, Hong Kong SAR, CRE Ref. No. 2019.250 (Submission Ref. No. NTEC-20190093).

This is a retrospective review of clinical data and no identifiable information is required. No patient consent to participate the study is required.

\section{Consent for publication}

All authors have read the manuscript and consent for its publication.

\section{Competing interests}

The authors declare that they have no competing interests relevant to this article.

\section{Author details}

'Department of Orthopedics \& Traumatology, Prince of Wales Hospital, Sha Tin, Hong Kong SAR, China. ${ }^{2}$ Present address: Department of Orthopedics \& Traumatology, Alice Ho Miu Ling Nethersole Hospital, Tai Po, Hong Kong SAR, China.

Received: 16 December 2020 Accepted: 29 March 2021

Published online: 12 June 2021

\section{References}

1. Ferket BS, Feldman Z, Zhou J, Oei EH, Bierma-Zeinstra SM, Mazumdar M (2017) Impact of total knee replacement practice: cost effectiveness analysis of data from the Osteoarthritis Initiative. BMJ. 356:j1131

2. Tang X, Wang S, Zhan S, Niu J, Tao K, Zhang Y, Lin J (2016) The prevalence of symptomatic knee osteoarthritis in China: results from the China Health and Retirement Longitudinal Study. Arthritis Rheum 68(3):648-653. https:// doi.org/10.1002/art.39465

3. Zhang Y, Xu L, Nevitt MC, Aliabadi P, Yu W, Qin M, Lui LY, Felson DT (2001) Comparison of the prevalence of knee osteoarthritis between the elderly Chinese population in Beijing and whites in the United States: The Beijing Osteoarthritis Study. Arthritis Rheum 44(9):2065-2071. https://doi.org/10.1 002/1529-0131 (200109)44:9<2065:AID-ART356>3.0.CO;2-Z

4. Odum SM, Troyer UL, Kelly MP, Dedini RD, Bozic KJ (2013) A cost-utility analysis comparing the cost-effectiveness of simultaneous and staged bilateral total knee arthroplasty. J Bone Joint Surg Am 95(16):1441-1449. https://doi.org/10.2106/JBJS.L.00373

5. Phillips JLH, Rondon AJ, Gorica Z, Fillingham YA, Austin MS, Courtney PM (2018) No difference in total episode-of-care cost between staged and simultaneous bilateral total joint arthroplasty. J Arthroplast 33(12):36073611. https://doi.org/10.1016/j.arth.2018.08.035

6. Wilkie W, Mohamed N, Remily E, Pastore M, Nace J, Delanois RE (2020) Same day versus staged total knee arthroplasty: do cost savings justify the risk? J Knee Surg. https://doi.org/10.1055/s-0040-1713734

7. Bohm ER, Molodianovitsh K, Dragan A, Zhu N, Webster G, Masri B, Schemitsch E, Dunbar M (2016) Outcomes of unilateral and bilateral total knee arthroplasty in 238,373 patients. Acta Orthop 87(Suppl 1):24-30. https://doi.org/10.1080/17453674.2016.1181817

8. Qutob M, Winemaker M, Petruccelli D, de Beer J (2013) Staged bilateral total knee arthroplasty: does history dictate the future? J Arthroplast 28(7):11481151. https://doi.org/10.1016/j.arth.2013.01.022

9. Bini SA, Khatod M, Inacio MC, Paxton EW (2014) Same-day versus staged bilateral total knee arthroplasty poses no increase in complications in 6672 primary procedures. J Arthroplast 29(4):694-697. https://doi.org/10.1016/j.a rth.2012.09.009

10. March LM, Cross M, Tribe KL, Lapsley HM, Courtenay BG, Cross MJ, Brooks PM, Cass C, Coolican M, Neil M, Pinczewski L, Quain S, Robertson F, Ruff S, Walter W, Zicat B, Arthritis C.O.S.T. Study Project Group (2004) Two knees or not two knees? Patient costs and outcomes following bilateral and unilateral total knee joint replacement surgery for OA. Osteoarthr Cartil 12(5):400-408. https://doi.org/10.1016/j.joca.2004.02.002

11. Sobh AH, Siljander MP, Mells AJ, Koueiter DM, Moore DD, Karadsheh MS (2018) Cost analysis, complications, and discharge disposition associated with simultaneous vs staged bilateral total knee arthroplasty. J Arthroplast 33(2):320-323. https://doi.org/10.1016/j.arth.2017.09.004

12. Leonard L, Williamson DM, Ivory JP, Jennison C (2003) An evaluation of the safety and efficacy of simultaneous bilateral total knee arthroplasty. J Arthroplast 18(8):972-978. https://doi.org/10.1016/S0883-5403(03)00282-1

13. Rikli RE, Jones CJ (1999) Functional fitness normative scores for communityresiding older adults, ages 60-94. J Aging Phys Act 7(2):162-181. https://doi. org/10.1123/japa.7.2.162

14. Reuben JD, Meyers SJ, Cox DD, Elliott M, Watson M, Shim SD (1998) Cost comparison between bilateral simultaneous, staged, and unilateral total joint arthroplasty. J Arthroplast 13(2):172-179. https://doi.org/10.1016/S08835403(98)90095-X

15. Malahias MA, Gu A, Adriani M, Addona JL, Alexiades MM, Sculco PK (2019) Comparing the safety and outcome of simultaneous and staged bilateral total knee arthroplasty in contemporary practice: a systematic review of the literature. J Arthroplast 34(7):1531-1537. https://doi.org/10.1016/j.arth.2019. 03.046

16. Zeni JA Jr, Snyder-Mackler L (2010) Clinical outcomes after simultaneous bilateral total knee arthroplasty: comparison to unilateral total knee arthroplasty and healthy controls. J Arthroplast 25(4):541-546. https://doi. org/10.1016/j.arth.2009.02.016

17. Fu D, Li G, Chen K, Zeng H, Zhang X, Cai Z (2013) Comparison of clinical outcome between simultaneous-bilateral and staged-bilateral total knee arthroplasty: a systematic review of retrospective studies. J Arthroplast 28(7): 1141-1147. https://doi.org/10.1016/j.arth.2012.09.023

18. Kulshrestha V, Kumar S, Datta B, Sinha VK, Mittal G (2018) Ninety-day morbidity and mortality in risk-screened and optimized patients undergoing two-team fast-track simultaneous bilateral TKA compared with unilateral TKA-A prospective study. J Arthroplast 33(3):752-760. https://doi.org/10.101 6/j.arth.2017.09.068

19. Sculco TP (2018) Simultaneous bilateral total knee arthroplasty: does it have a place ? Orthop Proc 99-B(Suppl 15):33

20. Bagsby D, Pierson JL (2015) Functional outcomes of simultaneous bilateral versus unilateral total knee arthroplasty. Orthopedics. 38(1):e43-e47. https:// doi.org/10.3928/01477447-20150105-59

21. Choi YJ, Lee HI, Ra HJ, Hwang DY, Kim TK, Shim SJ (2014) Perioperative risk assessment in patients aged 75 years or older: comparison between bilateral and unilateral total knee arthroplasty. Knee Surg Relat Res 26(4): 222-229. https://doi.org/10.5792/ksrr.2014.26.4.222

22. Memtsoudis SG, Ma Y, Gonzalez Della Valle A, Mazumdar M, Gaber-Baylis LK, Mackenzie CR et al (2009) Perioperative outcomes after unilateral and bilateral total knee arthroplasty. Anesthesiology. 111(6):1206-1216. https:// doi.org/10.1097/ALN.0b013e3181bfab7d 
23. Wong E, Nguyen CL, Park S, Parker D (2018) Simultaneous, same-anaesthetic bilateral total knee arthroplasty has low mortality and complication rates. Knee Surg Sports Traumatol Arthrosc 26(11):3395-3402. https://doi.org/10.1 007/s00167-018-4908-4

24. Hosick WB, Lotke PA, Baldwin A (1994) Total knee arthroplasty in patients 80 years of age and older. Clin Orthop Relat Res 299:77-80

25. Laskin RS (1999) Total knee replacement in patients older than 85 years. Clin Orthop Relat Res 367:43-49

26. Severson EP, Mariani EM, Bourne MH (2009) Bilateral total knee arthroplasty in patients 70 years and older. Orthopedics. 32(5):316-320. https://doi.org/1 0.3928/01477447-20090501-13

\section{Publisher's Note}

Springer Nature remains neutral with regard to jurisdictional claims in published maps and institutional affiliations.

Ready to submit your research? Choose BMC and benefit from:

- fast, convenient online submission

- thorough peer review by experienced researchers in your field

- rapid publication on acceptance

- support for research data, including large and complex data types

- gold Open Access which fosters wider collaboration and increased citations

- maximum visibility for your research: over $100 \mathrm{M}$ website views per year

At $\mathrm{BMC}$, research is always in progress.

Learn more biomedcentral.com/submissions 